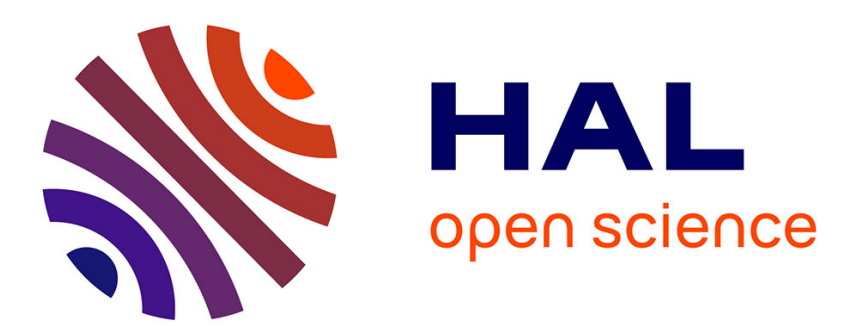

\title{
Towards Bandwidth and Energy Optimization in IEEE 802.15.4 Wireless Sensor Networks
}

\author{
Atmani Mouloud, Djamil Aissani, Yassine Hadjadj-Aoul
}

\section{To cite this version:}

Atmani Mouloud, Djamil Aissani, Yassine Hadjadj-Aoul. Towards Bandwidth and Energy Optimization in IEEE 802.15.4 Wireless Sensor Networks. Computing, 2017, pp.1-24. 10.1007/s00607-0170583-4 . hal-01657663

\section{HAL Id: hal-01657663 https://hal.inria.fr/hal-01657663}

Submitted on 7 Dec 2017

HAL is a multi-disciplinary open access archive for the deposit and dissemination of scientific research documents, whether they are published or not. The documents may come from teaching and research institutions in France or abroad, or from public or private research centers.
L'archive ouverte pluridisciplinaire HAL, est destinée au dépôt et à la diffusion de documents scientifiques de niveau recherche, publiés ou non, émanant des établissements d'enseignement et de recherche français ou étrangers, des laboratoires publics ou privés. 


\title{
Towards Bandwidth and Energy Optimization in IEEE 802.15.4 Wireless Sensor Networks
}

\author{
Mouloud Atmani · Djamil Aïssani . \\ Yassine Hadjadj-Aoul
}

Received: date / Accepted: date

\begin{abstract}
Transmission delay, throughput and energy are important criterions to consider in wireless sensor networks (WSN). In this way, IEEE 802.15.4 standard was conceived with the objective to reduce resource's consumption in both WSN and Personal Area Networks (WPAN). In such networks, the slotted CSMA/CA of still occupies a prominent place as a channel control access's mechanism with its inherent simplicity and reduced complexity.

In this paper, we propose to introduce a network allocation vector (NAV) as an extension of the fragmentation mechanism presented in [24] to reduce energy consumption and collisions in IEEE 802.15.4 networks. A Markov chain-based analytical model of the fragmentation mechanism, in a saturated traffic, is given as well as a model of the energy consumption using the NAV mechanism. The obtained results show that the fragmentation technique improves at the same time the throughput, the access delay and the bandwidth occupation. They, also, show that using the NAV allows reducing significantly the energy consumption when applying the fragmentation technique in slotted CSMA/CA under saturated traffic conditions.
\end{abstract}

Keywords IEEE 802.15.4 · slotted CSMA/CA · data fragmentation · NAV · Markov chains · energy consumption · bandwidth occupation

\section{Introduction}

Technological advances in miniaturization, coupled with improved longevity of wireless devices has led to the emergence of a keen interest, of both researchers and industrials, toward wireless sensor networks (WSN). A WSN is commonly

Mouloud Atmani · Djamil Aïssani

LaMOS Research Unit, University of Bejaia, Bejaia 06000, Algeria

E-mail: atmanimouloud@yahoo.fr

E-mail: djamil_aissani@hotmail.com

Yassine Hadjadj-Aoul

IRISA Laboratory, University of Rennes 1, France

E-mail: yhadjadj@irisa.fr 
composed by a huge number of inexpensive wireless communicating devices, denoted as nodes. These nodes have generally tight energy constraints imposed by the limited capacity of the batteries. Therefore, in WSN, the lifetime of the nodes is a key constraint to take into account; in addition to low transmission delay, good throughput and high-bandwidth utilization [1]. Thus, numerous protocols have been proposed in the literature to resolve these problems on the different layers of the protocol stack (Physical layer, MAC sub-layer, Network layer, ... ) [2].

The IEEE 802.15.4 standard, which is supported notably by ZigBee, is a technology defining the characteristics of the physical layer and the MAC sub-layer for low-rate, low-power and low-cost wireless personal area networks. The IEEE 802.15.4 standard has defined two access modes: the beacon mode and the nonbeacon mode. The latter considers the basic CSMA/CA mechanism, while the former considers the slotted CSMA/CA mechanism.

In the beacon mode, the one considered in this work, the coordinator sends regularly beacons to delimitate the superframe and to synchronize wireless sensors [3][4]. When using the slotted CSMA/CA access mode, the nodes randomly choose a backoff in the interval $\left[0,2^{B E}-1\right]$. The backoff is, then, decremented until reaching zero. At that time, the nodes compare the remaining time of the superframe and the transmission time of the ongoing packet. If the leftover time in the superframe is insufficient to complete the packet transmission, the transmission is deferred to the next superframe. This paper studied the drawback of deferred transmission in the IEEE 802.15.4 standard and provides an improved utilization of bandwidth. This problem is very little studied in the literature.

Pollin et al. proposed, in [5], a simple Markov chain model and an analytical evaluation of the slotted CSMA-CA mechanism of beacon enabled IEEE 802.15.4. In [6], the authors developed a generalized Markov chain model of the channel access mechanism. Compared to the Pollin's Markov chain, they took into account the retry limits' parameter in their model. In [7], Patro et al. proposed a modification of the slotted CSMA/CA protocol to use only one clear channel assessment (CCA1). The modified slotted CSMA/CA is modeled using Markov chains based on Pollin's model. The authors in [8] proposed to use three clear channel assessment. The additional carrier sensing (ACS) algorithm is introduced to enhance the carrier sensing mechanisms in the original slotted CSMA/CA. In [10], the authors proposed a Markov chains-based model, which considers the deferred transmission case. The analytical results show the effect of the transmission deferment on the network throughput.

In IEEE 802.11 standard, the fragmentation technique is implemented, and many studies have mentioned that this technique improves the network's throughput [12][13][14]. The authors, in [15], proposed a fragmentation mechanism for IEEE 802.15.4 wireless sensor network to improve the bandwidth utilization. The main issue in their protocol is the risk of collision. In fact, a collision occurs if a competitive node pulls a Backoff number equal to zero when the transmitter node attempts to send the remaining frame, in the beginning of the superframe.

In [24], we, particularly, focused our study on the deferred transmission problem in slotted CSMA/CA protocol. We have found that the period of deferment becomes larger as the packet length is large, which may cause a significant bandwidth loss. To avoid such loss of bandwidth, we have proposed to adapt the data fragmentation mechanism (already used in the IEEE 802.11 standard [12]) for the IEEE 802.15.4 standard. The adaptive data fragmentation assures the collision 
avoidance, when transmitting the remaining frame in case of fragmentation. Besides, we gave a particular attention, in our proposal, to keep the original operating principles of the slotted CSMA/CA mechanism.

This paper introduces the NAV used in the fragmentation mechanism in order to reduce the consumption of energy. Originally, the fragmentation mechanism proposed to improve the bandwidth occupation and the throughput. However, when a node chooses to transmit a remaining frame, other nodes may also attempt to transmit their data. This competition causes energy wastage. The proposed NAV solution allows informing the nodes willing to transmit about the completion of a data transmission. Thus, the nodes receiving the NAV stay in the idle state (i.e. backoff) for the remaining time, which allows them to conserve their energy.

The remainder of this paper is organized as follows. Section 2 gives an overview of the IEEE 802.15.4 standard and some related work proposed to improve slotted CSMA/CA protocol. Section 3 explains the NAV mechanism that we introduced in the data fragmentation mechanism in order to reduce the energy consumption. Section 4 shows the modelling of the fragmentation mechanism using Markov chains under saturated traffic and presents the energy consumption analysis using the NAV mechanism. Section 5 presents the analytical results of our solutions. Finally, we conclude and present future issues in section 6.

\section{Overview of IEEE 802.15.4}

The IEEE 802.15.4 standard defines the physical (PHY) layer and the MAC sublayer specifications for low-rate wireless personal area networks (LR-WPAN), which is the basis for many WSN such as ZigBee and 6LoWPAN [16].

The physical layer of the standard can operate in different frequency bands as follows: IEEE 802.15.4-2003 and IEEE 802.15.4-2006 operate in the $2450 \mathrm{MHz}$ band (16 channels), in the $915 \mathrm{MHz}$ band (10 channels ) and in the $868 \mathrm{MHz}$ band (1 channel). Other amendments of IEEE 802.15.4 have been proposed to take into account and support other bands: IEEE 802.15.4a (Ultra Wide Band), IEEE 802.15.4c (UWB and the China 779-787 MHz band), IEEE 802.15.4d (Japanese 950 - $956 \mathrm{MHz}$ band) and IEEE 802.15.4f (433 MHz band) [17][18][19][20].

The MAC sublayer of IEEE 802.15.4 defines many functions such as: beacon management, channel access mechanisms, guaranteed time slot (GTS) management, activation or not of acknowledged frame, devices association and disassociation with a PAN. Two channel access modes are supported in IEEE 802.15.4: Beacon-enabled mode and non-beacon mode. In beacon-enabled mode, the nodes use slotted CSMA/CA mechanism to access the channel. In non-beacon mode, the nodes use unslotted CSMA/CA.

In order to understand the structure of the superframe and the functioning of the CSMA/CA protocol, we must identify the key parameters with their numerical values, when possible. We consider the following physical characteristics: the frequency band of $2.4 \mathrm{Ghz}$ and the data rate of $250 \mathrm{kbps}$. In the IEEE 802.15.4 standard, the unit of measure used for most of the parameters is the symbol. Table 1 presents these parameters in unit of seconds or byte, knowing that: 1 symbol $=4$ bits [4].

The access channel periods, defining the superframe structure, are described by the PAN (Personal Area Network) coordinator. Indeed, the PAN coordinator 
Table 1 Parameters of the superframe and the slotted CSMA/CA protocol

\begin{tabular}{|c|c|c|c|}
\hline \multirow{14}{*}{ 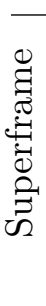 } & Parameter & Description & Value \\
\hline & $\mathrm{BO}$ & Beacon Order & $0 \leqslant B O \leqslant 14$ \\
\hline & & Superframe Order & $0 \leqslant S O \leqslant B O$ \\
\hline & aUnitBackoffPeriod & Duration of a Backoff period & $0.32 \mathrm{~ms}$ \\
\hline & aBaseSlotDuration & Duration of a time slot & $0.96 \mathrm{~ms}$ \\
\hline & aBaseSuperframeDuration & Minimum duration of the superframe & $15.36 \mathrm{~ms}$ \\
\hline & macMinLIFSPeriod & Duration of the Long IFS & $0.32 \mathrm{~ms}$ \\
\hline & macMinSIFSPeriod & Duration of the $s$ & $0.192 \mathrm{~ms}$ \\
\hline & aMaxSIFSFrameSize & The maximum size of frame followed by SIFS & 18 bytes \\
\hline & SIFS & Minimum short interframe space & $0.192 \mathrm{~ms}$ \\
\hline & LIFS & Minimum long interframe spacing & $1.28 \mathrm{~ms}$ \\
\hline & $\begin{array}{l}T_{a c k}-\text { wait } \\
\text { ACK }\end{array}$ & $\begin{array}{l}\text { Time to commence transmitting the ACK } \\
\text { Acknowledgment frame duration }\end{array}$ & $\begin{array}{l}0.192 \mathrm{~ms} \leqslant t_{\text {ack_wait }} \leqslant 1.024 \mathrm{~ms} \\
0.352 \mathrm{~ms}\end{array}$ \\
\hline & MAC & MAC header length & 7 bytes $\leq M A C<37$ bytes \\
\hline & PHY & Physical header length & 3bytes $\overline{\leq} M A C<21$ bytes \\
\hline \multirow{7}{*}{ 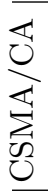 } & macMaxCSMABackoffs & $\begin{array}{l}\text { The maximum number that CSMA/CA was } \\
\text { required before declaring a channel access failure }\end{array}$ & 4 \\
\hline & macMinBE & Minimum number of the Backoff Exponent & 3 \\
\hline & macMaxBE & Maximum number of the Backoff Exponent & 5 \\
\hline & & Contention Window & 2 \\
\hline & macMaxCSMABackoffs & $\begin{array}{l}\text { The maximum number that CSMA/CA } \\
\text { is executed to attempt the channel access }\end{array}$ & 4 \\
\hline & $\mathrm{CCA}$ & Clear Channel Assessment & $0.128 \mathrm{~ms}$ \\
\hline & BLE & Battery Life Extension & $\{0,1\}$ \\
\hline
\end{tabular}

transmits in the beacon frames the following parameters: the Beacon Order (BO) and the Superframe Order (SO). The BO defines the beacon interval while the SO determines the superframe duration. The superframe duration, also called active portion of the superframe, is divided into two parts: Contention Access Period (CAP) and Contention Free Period (CFP). The details of the superframe structure are mentioned in figure 1.

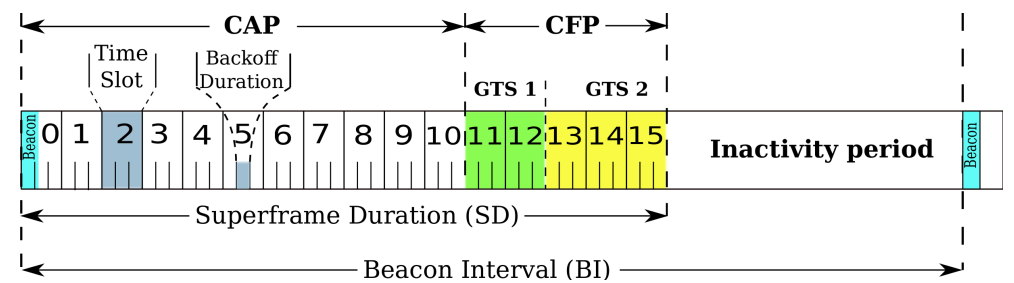

Fig. 1 Superframe structure in IEEE 802.15.4 standard

The value of the beacon interval (BI) and the superframe duration (SD) are determined respectively as follows:

$$
\begin{aligned}
& B I=\text { aBaseSuperframeDuration } * 2^{B O} . \\
& S D=\text { aBaseSuperframeDuration } * 2^{S O} .
\end{aligned}
$$

The values of aBaseSuperframeDuration, $B O$ and $S O$ are given in the table 1.

When, the value of $B O=15$, the PAN coordinator desactivates the sending of the beacon frame. The transmission of data frames by the nodes to the PAN coordinator are separated by an inter frame space (IFS). The IFS duration differs depending on the size of the transmitted frame. If a long frame ${ }^{1}$ (the frame length is greater than aMaxSIFSFrameSize) is transmitted, the separation is equal to a Long IFS (LIFS), otherwise, it is equal to a SIFS (see figure 2 for more details).

\footnotetext{
1 The concept of short and long frames already exists in the IEEE 802.15.4 standard
} 
Acknowledged transmission

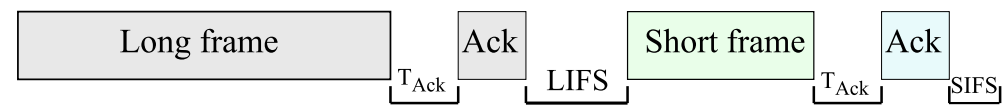

Unacknowledged transmission

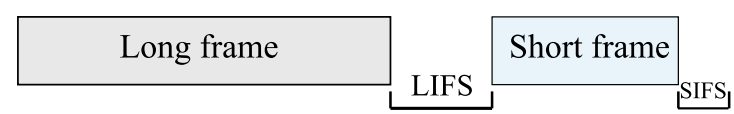

Fig. 2 IFS duration in ACK and Non ACK data transmission

\subsection{Slotted CSMA/CA mechanism}

In the CAP mode, the devices use the slotted CSMA/CA mechanism to get access to the wireless channel. To that purpose, three variables are considered: NB, $\mathrm{CW}$ and BE. NB, which is initialized to zero, represents the number of access tentatives for current transmission. CW represents the number of backoff periods during which the channel should be sensed idle before the communication can start. It is initialized to two. The backoff exponent (BE) helps in calculating the backoff delay. The initialization of the BE depends on the value of the Battery Life Extension (BLE) received in the beacon frame. If the BLE is equal to zero, the $\mathrm{BE}$ is initialized to macMinBE (i.e. equal to three by default). If it is equal to one, the $\mathrm{BE}$ is initialized to the lesser value between 2 and macMinBE.

Once the three variables are initialized, the node waits during a random period of Backoff chosen in the range $\left[0,2^{B E}-1\right]$. If the pulled number is greater than the remaining number of backoff periods in the CAP, the MAC sublayer pauses the backoff countdown at the end of the CAP and resume it at the start of the CAP in the next superframe.

At the expiration of the random backoff delay, the MAC sublayer shall ensure that the remaining CSMA/CA operations can be undertaken and the entire transaction (two CCA "Clear Channel Assessment", the frame transmission, and the acknowledgment) can be completed before the end of the CAP. Two cases are possible:

\section{Case 1: The remaining time in the CAP is sufficient:}

The MAC sublayer requests to the physical layer to perform two CCA:

1. The channel is assessed to be busy during one of the CCA: both NB and $\mathrm{BE}$ are incremented by one (BE shall be no more than macMaxBE), CW is reset to two. If $\mathrm{NB}$ is less than or equal to macMaxCSMABackoffs (4 by default), a new $\mathrm{BE}$ is pulled randomly in the range $\left[0,2^{B E}-1\right]$. If $\mathrm{NB}$ is greater than macMaxCSMABackoffs, the CSMA/CA algorithm shall terminate with a channel access failure status.

2. The channel is assessed to be idle during the first CCA: CW is decremented by one and then determine whether it is equal to zero.

The same procedure is considered for the second CCA. If CW is equal to zero, the data frame is transmitted. 
Case 2: The remaining time in CAP is insufficient:

The transmission will be deferred to the next superframe and the new CSMA/CA is executed at the beginning of the CAP (read [4] for more precisions).

\subsection{Deferred transmission problems in IEEE 802.15.4}

In slotted CSMA/CA mechanism, as described in IEEE 802.15.4-2003 [3], when a node defers its transmission to the next superframe, it performs a CCA in the new superframe. In this case, a problem of data collision may occur when more nodes defer their transmissions. To resolve the problem of collision due to deferred transmissions a revised version of the IEEE 802.15.4-2003 standard is published in IEEE 802.15.4 version 2006. In the revised version, the bandwidth occupation problem, caused by the deferred transmission, is not resolved.

The deferred transmission is analysed in [9] to study the effect of deferments on the performance of WSN. The first solution to improve bandwidth occupation is given in [15]. In this paper, the authors proposed to introduce the data fragmentation in IEEE 802.15.4. This solution presents, however, the risk of collisions at the beginning of superframes after data fragmentation.

To eliminate the problem of collision in data fragmentation, we proposed, in [24], an improvement of the classical fragmentation mechanism. The improvement consists in favoring the completion of the transmission of the remaining frame in the next superframe for the node, which just sent a fragment, in a way to improve bandwidth occupation.

\section{Proposed fragmentation with NAV mechanisms for IEEE 802.15.4}

Considering the bandwidth occupation problem caused by the deferred transmission in slotted CSMA/CA, in this section, we describe briefly the fragmentation mechanism (the details can be found in [24]) and its enhancement by the NAV technique that we introduced in order to improve energy conservation in IEEE 802.15.4.

\subsection{Fragmentation solution for the IEEE 802.15.4}

The main idea of the fragmentation mechanism consists in splitting the original frame (long frame) into two parts: the short frame of 18 bytes maximum ${ }^{2}$ and the remaining frame as depicted in the figure 3.

We propose to introduce the fragmentation mechanism in slotted CSMA/CA protocol as a solution to improve bandwidth occupation. Thus, if a node has a long frame to transmit, and the remaining time (RT) in the CAP is insufficient, the new MAC sublayer check if RT is larger to finish the entire transaction with a short frame. If it is the case, the CCA is performed and the fragmentation is applied. The node transmits a short frame in the current superframe and reserves the start of the new superframe to transmit the remaining frame, as shown in figure 4 .

\footnotetext{
${ }^{2}$ IEEE 802.15.4 standard defined the biggest length of the short and the long frames.
} 


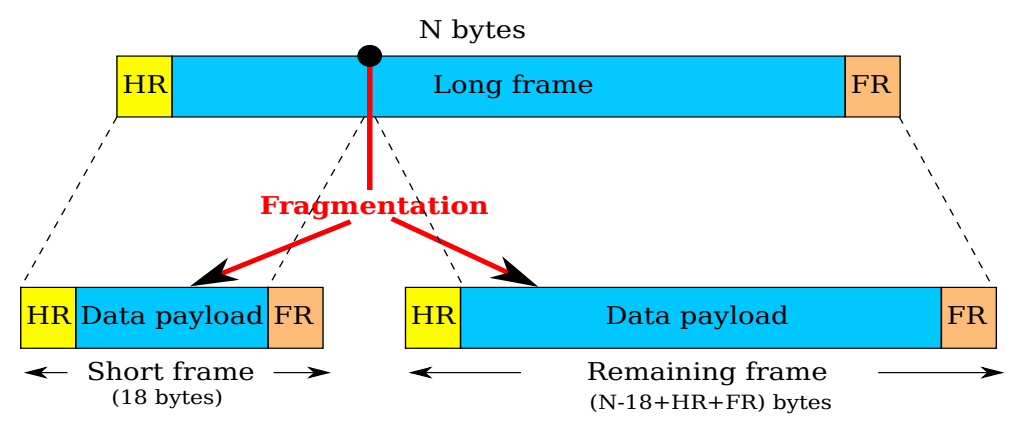

Fig. 3 Fragmentation mechanism in our contribution

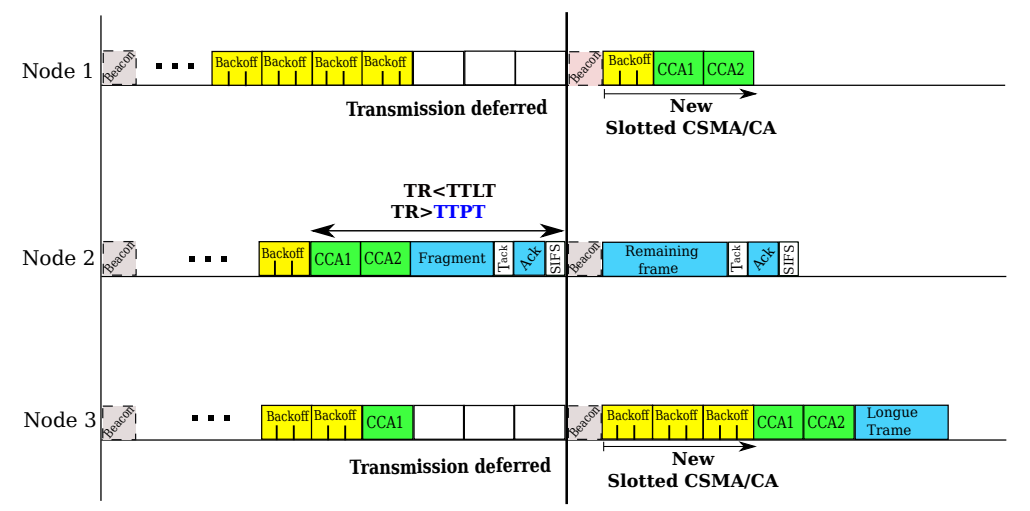

Fig. 4 Fragmentation mechanism proposed for IEEE 802.15.4

The fragmentation technique described here attempts to reduce the wastage bandwidth, collision and improve the network throughput. Effectively, the data fragmentation mechanism can improve the performances of IEEE 802.15.4 network in terms of bandwidth occupation. However, when using data fragmentation, the energy consumption is not improved. We observe, in figure 4 , that all nodes in the WPAN, except the node transmitting the data fragment, attempt to access the channel by executing the slotted CSMA/CA algorithm. This leads to a wastage of energy. Indeed, the fragmentation mechanism proposed in [11][24] favors the sensor that transmitted the data frame in the previous superframe to continue by sending the leftover frame in the next superframe.

\subsection{NAV-based energy conservation solution}

To improve the performances of the fragmentation mechanism that we have described in [24], we have introduced the NAV technique. As we can see in figure 5 , only the node that have been transmitted the data fragment can access the channel in the beginning of the next superframe.

The NAV information will be transmitted in the beacon frame. Hence, all the nodes in the PAN turn off their radio module during the NAV period to conserve their energy, excepting the transmitter of the data fragment. 


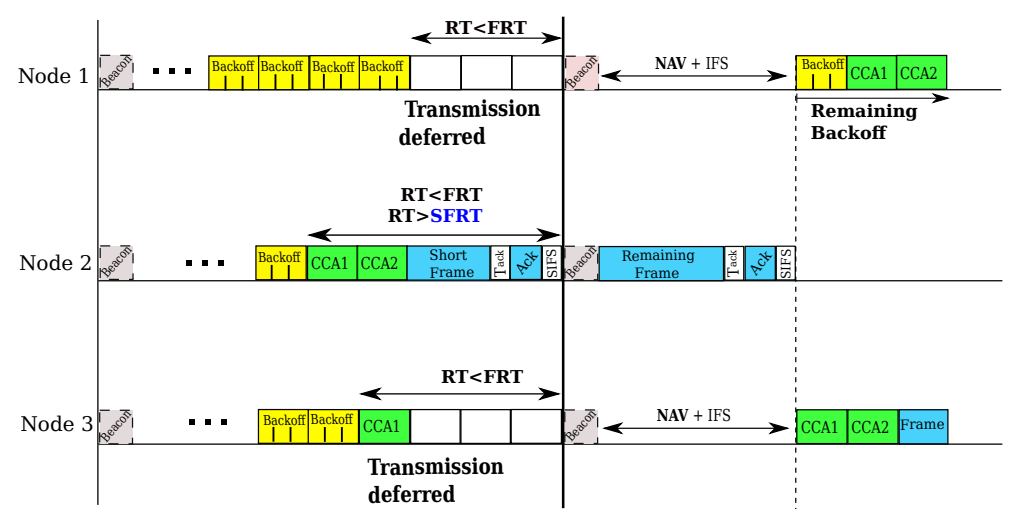

Fig. 5 Solution to improve bandwidth occupation in IEEE 502.15.4

\section{Analytical model of IEEE 802.15.4 with fragmentation}

In this section, we present the analytical model of the adaptive data fragmentation mechanism in the slotted CSMA/CA protocol and its analysis. We consider that the network is composed by $n$ nodes connected to the PAN coordinator forming a star topology, with assumptions of saturated traffic and an ideal channel.

To show the benefit of the fragmentation mechanism in the IEEE 802.15.4 wireless sensor networks, we, firstly, model the slotted CSMA/CA protocol with the data fragmentation mechanism to study the behavior of a single node. The proposed Markov chain model (see figure 6) allows us to calculate the stationary probability that a node attempts the first carrier sensing, noted $\tau$, in the steady state. Secondly, we express the performance parameters of the fragmentation mechanism based on $\tau$.

Table 2 gives the probabilities used in the Markov chain and the performance parameters.

Table 2 Analytical model probabilities

\begin{tabular}{l|l}
\hline Probability & Description \\
\hline$\tau$ & Probability to attempt a first carrier sensing \\
$\alpha$ & Probability to find a channel busy in CCA1 \\
$\beta$ & Probability to find a channel busy in CCA2 \\
$P_{d}$ & Probability to defer a transmission \\
$P_{f}$ & Probability to fragment a data frame \\
$P_{t} r$ & Transmission probability \\
$P_{s}$ & Successful transmission probability \\
$D_{P r o b}$ & Overall deferment probability \\
\hline
\end{tabular}

We study, in the following, the behavior of this node over time.

Let $S(t), B(t)$ and $T(t)$ be the stochastic processes representing, respectively, the backoff stage, the state of the backoff counter and the type of the data frame 
to be transmitted at time $t$. Their respective values are given as follows:

$$
\begin{aligned}
& S(t)=(0 . . m) . \\
& B(t)=\left(-2 . . W_{i}-1\right) . \\
& T(t)=\{-1,0,1,2\} .
\end{aligned}
$$

where $m=\operatorname{macMax} C S M A B a c k o f f s, W_{i}=2^{i} * W_{0}$, and $W_{0}=2^{B E}-1$. Note that, the backoff counter $B(t)$ is divided into two periods: the backoff period pulled between $\left[0,2^{B E}\right]$ and the two clear channel assessment (CCA) periods (i.e., -2 and $-1)$

The tuple $\{S(t), B(t), T(t)\}$ is a three dimensional Markov chain, which is depicted in figure 6 .

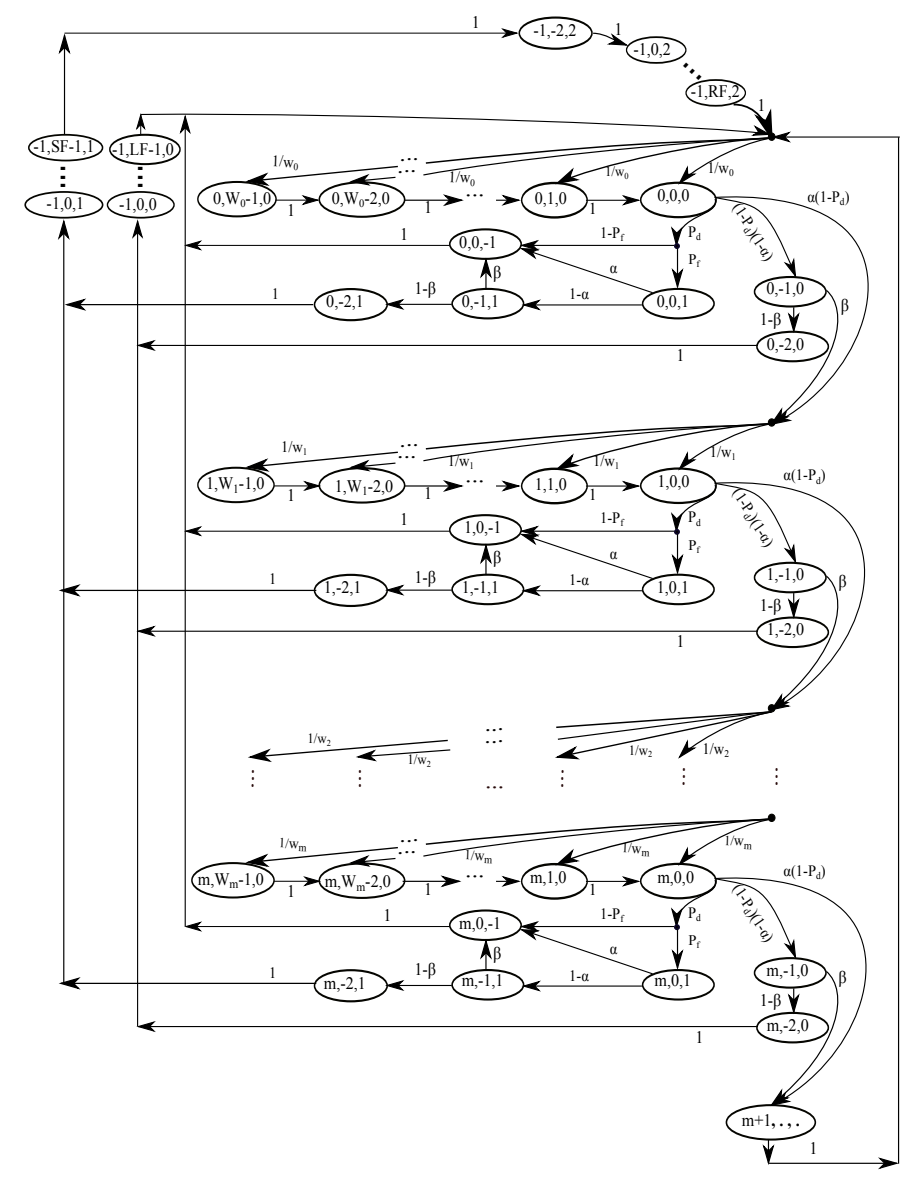

Fig. 6 Markov chain of slotted CSMA/CA with an adaptive data fragmentation mechanism 
The states $(i,-1, j)$ and $(i,-2, j)$ represent the sensing periods: CCA1 and CCA2, respectively. The states $(i, k, 0),(i, k, 1),(i, k, 2)$ and $(i, k,-1)$ denote, respectively, that: the node have a data frame to transmit, the data frame is fragmented to a short frame which will be transmitted in the current superframe, the node have a remaining frame to be transmited in the next superframe, and the node defers the transmission of the original frame to the next superframe. Note that $\tau$ of each node is independent from the other nodes.

The transition probabilities associated with the Markov chain are:

$$
\begin{aligned}
P(i, k, 0 \mid i, k+1,0)= & 1, \quad k \leqslant 0 . \\
P(i,-1, j \mid i, 0, j)= & (1-\alpha)\left[\left(1-P_{d}\right)+P_{d} P_{f}\right], \\
& j \in\{0,1\}, \quad i \leqslant m . \\
P(i,-2, j \mid i,-1, j)= & 1-\beta, \quad j=\{0,1\}, \quad i \leqslant m . \\
P(i, k, j \mid i-1,0, j)= & \left(1-P_{d}\right) \frac{\alpha+(1-\alpha) \beta}{W_{i}}, \quad i \leqslant m . \\
P(0, k, j \mid i, 0, j) & =\frac{P_{d}}{W_{0}}\left[\left(1-P_{f}\right)+P_{f}[\alpha+(1-\alpha) \beta]\right], \\
& j \in\{0,1\}, \quad i \leqslant m . \\
P(i, 0,1 \mid i, 0,0) & =P_{d} P_{f}, \quad i \leqslant m .
\end{aligned}
$$

Equation (1) defines the decrement probability of the backoff counter. Equations (2) and (3) describe the probability to find the channel idle for the first clear sense (CCA1) and the second clear sense (CCA2), respectively. Equation (4) denotes a busy channel, in which a node selects a new backoff delay in the new stage. Equation (5) represents the probability to defer the transmission to the next superframe when the remaining time in the CAP is too small to transmit a packet (i.e., even a fragmented packet) or the fragmentation is possible but the channel is busy. Equation (6) describes the possibility of fragmentation when the remaining delay in the CAP is too small to assure a successful transmission of the original frame.

Let $b_{i, k, j}=\lim _{t \rightarrow+\infty} P\{S(t)=i, B(t)=k, T(t)=j\}$, be the stationary distribution of our Markov chain. Basing on the equation (4), the probability that a node selects a new state in the upper stage, when the channel is sensed busy during CCA1 or CCA2, is calculated as follows:

$$
b_{i, 0,0}=\left(1-P_{d}\right)[\alpha+(1-\alpha) \beta] b_{i-1,0,0}, i \in(1, m),
$$

which can be generalized to:

$$
b_{i, 0,0}=\left[\left(1-P_{d}\right)[\alpha+(1-\alpha) \beta]\right]^{i} b_{0,0,0}, i \in(1, m) .
$$

The probability to be in any state of the first stage can be given as follows:

$$
\begin{aligned}
b_{0, k, 0}= & \frac{W_{0}-k}{W_{0}}\left[\left[(1-\alpha)(1-\beta)\left(1-P_{d}\right)\right] \sum_{i=0}^{m} b_{i, 0,0}\right. \\
& \left.+\left(1-P_{d}\right)[\alpha+(1-\alpha) \beta] b_{m, 0,0}\right]
\end{aligned}
$$


The probability to be in any backoff state in any stage is given by:

$$
b_{i, k, 0}=\frac{W_{i}-k}{W_{i}} b_{i, 0,0}, i \in(1, m), k \in\left(1, W_{i}-1\right) .
$$

By imposing the normalization condition, to compute the probability that a node be in the state $b_{0,0,0}$, the sum of the probabilities is equal to 1 :

$$
\begin{aligned}
1= & \sum_{i=0}^{m} \sum_{k=0}^{W_{i}-1} b_{i, k, 0}+\sum_{i=0}^{m} \sum_{k=-2}^{-1} b_{i, k, 0}+\sum_{i=0}^{m} \sum_{k=-2}^{0} b_{i, k, 1}+\sum_{i=0}^{m} b_{i, 0,-1} \\
& +\sum_{k=0}^{L F-1} b_{-1, k, 0}+\sum_{k=0}^{S F-1} b_{-1, k, 1}+\sum_{k=0}^{R F-1} b_{-1, k, 2} .
\end{aligned}
$$

Each parameter of equation (11) is described in the following equation:

$$
\begin{aligned}
\sum_{i=0}^{m} \sum_{k=0}^{W_{i}-1} b_{i, k, 0} & =\sum_{i=0}^{m} \sum_{k=1}^{W_{i}-1} b_{i, k, 0}+\sum_{k=0}^{W_{i}-1} b_{0, k, 0} \\
& =\frac{1}{2}\left[\frac{1-(2 x)^{(m+1)}}{1-2 x} W_{0}+\frac{1-x^{(m+1)}}{1-x}\right] b_{0,0,0}
\end{aligned}
$$

Where, $x=\left(1-P_{d}\right)[\alpha+(1-\alpha) \beta]$.

$$
\begin{gathered}
\sum_{i=0}^{m} \sum_{k=-2}^{-1} b_{i, k, 0}=(1-\alpha)(2-\beta)\left(1-P_{d}\right) \sum_{i=0}^{m} b_{i, 0,0}, \\
\sum_{i=0}^{m} \sum_{k=-2}^{0} b_{i, k, 1}=[1+(1-\alpha)(2-\beta)] P_{f} P_{d} \sum_{i=0}^{m} b_{i, 0,0}, \\
\sum_{i=0}^{m} b_{i, 0,-1}=\left[(\alpha+(1-\alpha) \beta) P_{f} P_{d}+\left(1-P_{f}\right) P_{d}\right] \sum_{i=0}^{m} b_{i, 0,0}, \\
\sum_{k=0}^{L F-1} b_{-1, k, 0}=(1-\alpha)(1-\beta)\left(1-P_{d}\right) L F \sum_{i=0}^{m} b_{i, 0,0}, \\
\sum_{k=0}^{S F-1} b_{-1, k, 1}=(1-\alpha)(1-\beta)\left(1-P_{d}\right) P_{f} P_{d} S F \sum_{i=0}^{m} b_{i, 0,0}, \\
\sum_{k=0}^{R F-1} b_{-1, k, 2}=(1-\alpha)(1-\beta) P_{f} P_{d}(S F+R F) \sum_{i=0}^{m} b_{i, 0,0}, \\
b_{-1,-2,2}=(1-\alpha)(1-\beta) P_{f} P_{d} \sum_{i=0}^{m} b_{i, 0,0} .
\end{gathered}
$$


Equation (11) can, thus, be simplified as follows:

$$
\begin{aligned}
1= & {\left[\left[\frac{1}{2}+P_{d}\left[1+P_{f}(2-\alpha)\right]+(1-\alpha)\left(1-P_{d}\right)+(1-\alpha)(1-\beta)\left(1-P_{d}\right)(1+L F)\right.\right.} \\
& \left.\left.+(1-\alpha)(1-\beta) P_{f} P_{d}(2 S F+R F)\right] \frac{1-x^{(m+1)}}{1-x}+\frac{1-(2 x)^{(m+1)}}{2(1-2 x)} W_{0}\right] b_{0,0,0}
\end{aligned}
$$

From equation (20), we can express the probability $b_{0,0,0}$. Then, we can express the probability $\tau$ as follows:

$$
\begin{aligned}
& \tau=\sum_{i=0}^{m} b_{i, 0,0} \\
& \tau=\left[\frac{1}{\left[P _ { d } \left[1+P_{f}[(2-\alpha)+(1-\alpha)(1-\beta)(2 S F+R F)]+(1-\alpha)\left[\left(1-P_{d}\right)\right.\right.\right.}\right. \\
& \left.\overline{\left.+(1-\beta)(1+L F)]+\frac{1}{2}\right] \frac{1-x^{m+1}}{1-x}+\frac{1-(2 x)^{m+1}}{2(1-2 x)} W_{0}}\right] \frac{1-x^{m+1}}{1-x} .
\end{aligned}
$$

The computation of the probability $\tau$ depends mainly on the following probabilities:

A) Probability $\alpha$ : is the probability to find the channel busy during CCA1 due to a data frame transmission. Similarly to [5], we express this probability as follows:

$$
\alpha=L\left[1-(1-\tau)^{n-1}\right](1-\alpha)(1-\beta) .
$$

Where, $L$ and $n$ represent the number of time slots occupied by the successful transmission of the data frame and the number of nodes, respectively.

B) Probability $\beta$ : is defined as the probability to find the channel busy in the CCA2, given that it is free in the CCA1 period.

$$
\beta=\frac{1-(1-\tau)^{n-1}}{2-(1-\tau)^{n}} .
$$

C) Probability $P_{d}$ : this probability introduces the deferment of the transmission when the remaining time in the CAP period is not sufficient to complete the transmission (i.e. time $>t_{i}$, see figure 7 ). The deferment probability is given by:

$$
P_{d}=\frac{L F+C C A 1}{C A P} \text {. }
$$

D) Probability $P_{f}$ : is the probability of fragmentation. $P_{f}$ is the probability to find sufficient time in the CAP period to sense two CCAs (i.e., CCA1 and CCA2) and to transmit a short frame when there is not enough time in the CAP to complete a long frame transmission. figure 7 shows that a short frame can be transmitted if the current time in the CAP is in the blue stripes part (i.e, time $\left.\in] t_{i}, t_{j}\right]$ ). On the opposite, no transmission will be deferred to the next superframe, if the time is in the red stripes (i.e, time $>t_{j}$ ) part. 


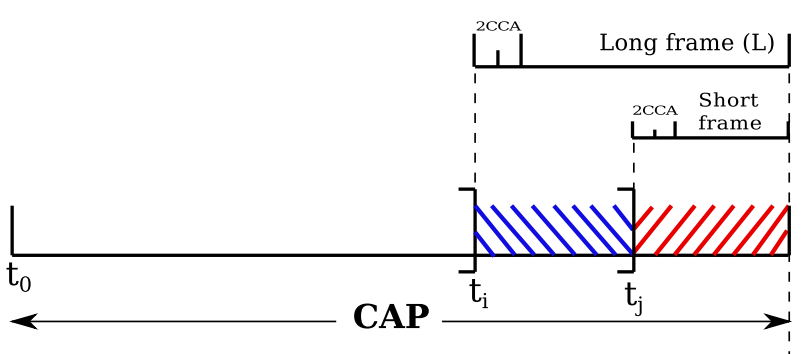

Fig. 7 Required time of short and long frames

$$
P_{f}=\frac{L F-S F}{C A P}
$$

Where, SF represents the length of the short frame.

\subsection{Saturation throughput}

The saturation throughput (noted $N_{T h}$ ), as defined in [21], is the fraction of time that the channel is used to successfully transmit the data frame. Therefore, $N_{T h}$ depends, on the following probabilities:

A) Transmission probability $\left(P_{t r}\right)$ : represents the probability that at least one node (among $n$ nodes) is in the beginning of the first clear sensing (CCA1) with probability $\tau$, the channel sensed free in CCA1 and CCA2 and the transmission will not be deferred.

$$
P_{t r}=\left[1-(1-\tau)^{n}\right](1-\alpha)(1-\beta)\left(1-P_{d}\right) .
$$

B) Successful transmission probability $\left(P_{s}\right)$ : is the probability that exactly one transmission occurred in the channel, conditioned by the transmission probability (as defined in [21]).

$$
P_{s}=\frac{n \tau(1-\tau)^{n-1}(1-\alpha)(1-\beta)\left(1-P_{d}\right)}{P_{t r}} .
$$

Now, we can express the saturation throughput as follows:

$$
N_{t h}=\frac{P_{t r} P_{s} T_{\text {pload }}}{\left(1-P_{t r}\right) \sigma+P_{t r} P_{s} T_{s}+\left(1-P_{s}\right) T_{c}+P_{d}\left(1-P_{f}\right) T_{D e f}} .
$$

Where, $T_{\text {pload }}$ is the time occupied by the packet transmission, $\sigma$ is the duration of an empty time slot, $T_{s}$ is the time of a successful transmission of a packet, $T_{c}$ is the time during which the channel is busy due to a collision and $T_{D e f}$ the average time wasted when deferring a transmission.

$$
\left\{\begin{array}{rl}
T_{s}= & T_{P H Y}+T_{M A C}+T_{\text {pload }}+2 T_{C C A}+T_{L I F S} \\
& +T_{\text {ack_wait }}+T_{A C K} . \\
T_{c}=\quad & T_{P H Y}+T_{M A C}+T_{\text {pload }}+2 T_{C C A}+T_{L I F S} \\
& +T_{a c k \_w a i t} . \\
T_{\text {Def }}= & \frac{T_{S F}+T_{P H Y}+T_{M A C}+T_{S I F S}+2 T_{c c a}-\varepsilon}{2}
\end{array} .\right.
$$


where $\varepsilon$ indicates that there is not sufficient time to complete the short frame transmission in the current superframe.

\subsection{Deferment probability}

We express, in this subsection, the overall probability to defer the transmission because of insufficient time in the CAP. Note that, like most of the existing models of the IEEE 802.15.4 standard, we have not considered the remaining time before attempting to access the channel.

In our solution, the transmission will be deferred only if there is not sufficient time to transmit a short frame. $D_{\text {Prob }}$ represents the probability that there is at least one node in the beginning of the first clear sensing (CCA1) and found that the remaining time is insufficient to complete the transmission nor the long neither the short frames. According to the CSMA/CA protocol, the evaluation of the leftover time in the CAP period is the first condition to be checked before beginning to sense the channel during 2 CCAs. Thus, the probability $D_{\text {Prob }}$ can be expressed as follows:

$$
D_{\text {Prob }}=\left[1-(1-\tau)^{n}\right] P_{d}\left(1-P_{f}\right)
$$

\subsection{Deferment average time}

The total transmission delay can be caused by: the node processing delay, the queuing delay, the access delay, and the propagation delay [22]. In our work, we are interested by calculating the deferment average time (i.e. $D A T$ ). The deferment delay is a part of the access delay. It depends on the packet length to be transmitted and the remaining time on the CAP. The expected value of $D A T$ is:

$$
D A T=D_{P r o b} T_{D e f}
$$

We can note that, when the packet length increases, the time wasted in deferring transmission increases $\left(T_{D e f}\right)$, therefore, the bandwidth utilization decreases.

\subsection{Access delay}

The access delay includes the following times: the successful transmission, the collisions, the backoff period, the clear sensing and the time of deferring a transmission. The access delay $A D$ of the successfully transmitted packet is defined in [22] as follows:

$$
A D=\frac{\text { Duration of one packet }}{\text { Fraction of time of a successful transmission }}
$$

The duration of one packet is the time spent to transmit a packet. The fraction of time of a successful transmission (FTST) can be calculated as follows:

$$
F T S T=\frac{P_{t r} P_{s} T_{s}}{E\left[E\left[S_{i}\right]+2 T_{c c a}\right]+T_{D e f}+P_{t r} P_{s} T_{s}+\left(1-P_{s}\right) T_{c}} .
$$


where, $E\left[S_{i}\right]$ represents the average backoff counter in the backoff stage i $\left(S_{i}=\right.$ $\left.w_{i} / 2\right)$. Therefore, $E\left[E\left[S_{i}\right]\right]$ be the average backoff in all $m+1$ stages.

It can be calculated as:

$$
E\left[E\left[S_{i}\right]+2 T_{c c a}\right]=\frac{\sum_{i=0}^{m} E\left[S_{i}\right] 2 T_{c c a}}{m+1} .
$$

So, $A D$ can be expressed as:

$$
A D=\frac{T_{P H Y}+T_{M A C}+T_{\text {pload }}}{F T S T}
$$

\subsection{Energy consumption}

In order to analyze the energy consumption when introducing the NAV technique in IEEE 802.15.4 with fragmentation, we calculate only the energy consumed for the data transmission $E_{\text {data_trans. }}$. As discussed above, the NAV technique is introduced to reduce the energy consumption in the fragmentation process, especially in the transmission of the remaining frame.

Let's define the energy consumed to transmit the original frame as $E_{F}$, the energy consumed to transmit the short frame or the fragment as $E_{S F}$ and the energy consumed to transmit the remaining frame as $E_{R F}$. The transmission energy is given as follows:

$$
E_{\text {data_trans }}=\left(1-P_{f}\right) E_{F}+P_{f}\left(E_{S F}+E_{R F}\right) .
$$

We compare our solution with the simple fragmentation technique proposed in [24].

A) Energy of IEEE 802.15.4 with fragmentation

$$
\begin{gathered}
E_{F}=\frac{N}{2} \tau\left(1-P_{d}\right)\left[(1-\alpha)(1-\beta) P_{s} E_{t x}+(1-\alpha) \beta 2 E_{c c a}\right. \\
\left.+\alpha E_{c c a}+(1-\alpha)(1-\beta)\left(1-P_{s}\right) E_{c o l l}\right] . \\
E_{S F}=P_{d} \tau(1-\alpha)(1-\beta) P_{s} E_{t x} . \\
E_{R F}=\frac{N-1}{2}\left[E_{m c u}+\tau \alpha E_{c c a}\right]+E_{t x} .
\end{gathered}
$$

In this case, when a node have transmitted a short frame (data fragment) in the previous superframe, it is favored to access the channel in the beginning of the next superframe. However, other nodes may attempt to access the channel and consume computational energy $\left(E_{m c u}\right)$ and the energy of clear channel assessment $\left(E_{c c a}\right)$. 
B) Energy of IEEE 802.15.4 with fragmentation and NAV

The energy consumed to transmit the original frame $E_{F}$ and the short frame $E_{S F}$ is the same that 38 and 39 respectively.

$$
E_{R F}=(N-1) E_{i d l e}+E_{t x} .
$$

Where, $E_{t x}$ is the energy consumed to transmit a data frame (original frame, short frame or remaining frame).

$$
\begin{aligned}
E_{t x}= & T_{t a} R_{t a}+2 T_{c c a} R_{r x}+T_{p l o a d} R_{t x}+\left(T_{H R}+T_{F R}\right) R_{t x} \\
& +T_{t a} R_{t a}+T_{a c k \_w a i t} R_{r x} . \\
E_{c c a}= & 2 T_{t a} R_{t a}+T_{c c a} R_{r x} . \\
E_{c o l l}= & 2 T_{t a} R_{t a}+2 T_{c c a} R_{r x}+T_{p l o a d} R_{t x}+\left(T_{a c k \_w a i t}+T_{A C K}\right) R_{r x} . \\
E_{\text {idle }}= & 2 T_{t a} R_{t a}+T_{R F} R_{i d l e} . \\
E_{m c u}= & T_{t a} R_{t a}+\frac{\left(T_{R F}-T_{c c a}\right)}{2} R_{m c u} .
\end{aligned}
$$

The numerical values of different radio state in terme of power consumption are presented in the table 3 . These values are inspired from the paper [25].

Table 3 Numerical values of radio power consumptions

\begin{tabular}{l|l|l}
\hline Variable & Description & value \\
\hline$R_{t x}$ & Radio transmission power & $48 \mathrm{mw}$ \\
$R_{r x}$ & Radio reception power & $56.5 \mathrm{mw}$ \\
$R_{t a}$ & Radio transient to actif power & $35 \mathrm{mw}$ \\
$R_{i d l e}$ & Radio idle power & $2.79 \mathrm{mw}$ \\
$R_{m c u}$ & Radio micro controller unit power & $1.5 \mathrm{mw}$ \\
\hline
\end{tabular}

\section{Performance evaluation}

In this section, we evaluate the performance of the proposed solution in improving the throughput, the access time for successful transmissions and the bandwidth utilization. To ensure the comparability of our solution with the IEEE 802.15.4 standard, we have modeled this latter by taking into account the deferment of the transmission when the remaining time in the CAP is not sufficient to complete the transmission of a frame.

In our work, we used the default parameters as summarized in tables 1 and 4 according to IEEE 802.15.4 standard.

In the results that we will address bellow, we present for each performance parameter two figures according to the $B L E$ parameter $(B E=2$ or 3$)$ as defined by the IEEE 802.15.4 standard.

\section{A) Deferment transmission}

In figure 8, we present the probability to defer the transmission when there is not enough time in the CAP period to complete the transmission of packet. In this 
Table 4 IEEE 802.15.4 Physical and MAC parameters

\begin{tabular}{l|l}
\hline Parameter & Value \\
\hline frequency band & $2.4 \mathrm{Ghz}$ \\
Data rate & $250 \mathrm{kbps}$ \\
$T_{\text {pload }}$ & variable \\
MAC & 16 bytes \\
PHY & 6 bytes \\
\hline
\end{tabular}

figure, we show that when the length of the packet is just greater to the short frame $(L=3$ slots and shortframe $=2$ slots $)$, the probability of deferment is not high. On the contrary, when the packet length increases, the fragmentation mechanism is interesting to avoid the deferring of the transmission.

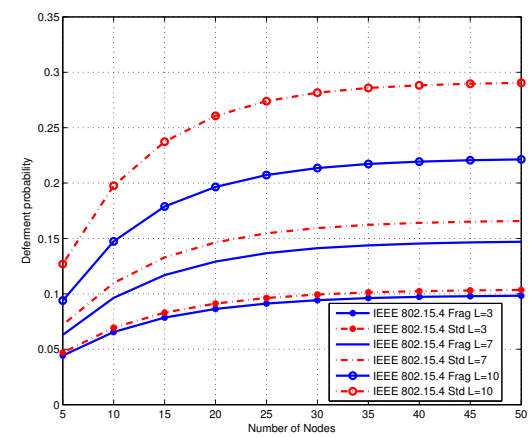

(a) $\mathrm{SO}=0$ and $\mathrm{BE}=2$

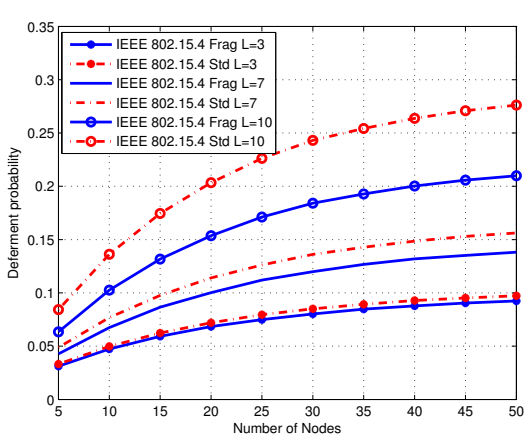

(b) $\mathrm{SO}=0$ and $\mathrm{BE}=3$

Fig. 8 Deferment probabilities of the transmission for $\mathrm{BO}=\mathrm{SO}=0$, parameterized on different $\mathrm{BE}$ and $\mathrm{L}$

In this figure, we show that when the length of the frame is just greater to the short frame ( $L=3$ slots and shortframe $=2$ slots $)$, the probability of deferment is not high. However, when the frame length increases, the fragmentation mechanism is interesting to avoid the transmissions' deferment. There is also a slight difference between subfigures 8(a) and 8(b) about the values of BE. We see that, if the network is saturated, a bigger BE value allows improving the obtained results.

\section{B) Saturation throughput}

The saturation throughput versus different numbers of nodes are shown in figure 9. We see, in subfigures 9 (a) and 9(b), that the fragmentation mechanism improves the network throughput.

The results presented in [23] is similar to ours. Note, however, that the authors did not consider the time wasted by the deferment of the transmission which reduces the network throughput.

\section{C) Deferment average time}

Figure 10 depicts the wastage of time due to transmissions' deferment. The obtained results clearly confirms the benefit of adopting fragmentation in IEEE 


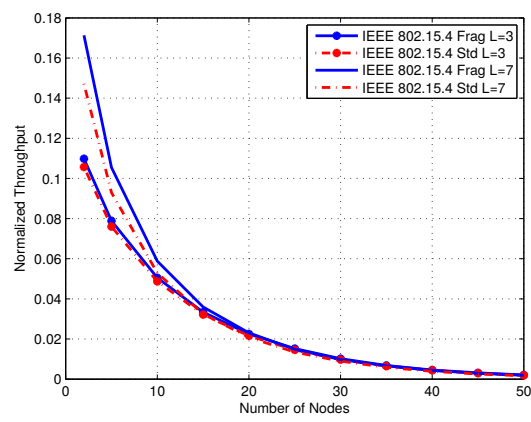

(a) $\mathrm{SO}=0$ and $\mathrm{BE}=2$

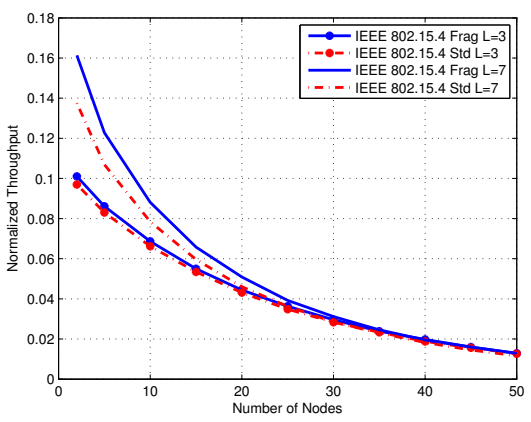

(b) $\mathrm{SO}=0$ and $\mathrm{BE}=3$

Fig. 9 Saturation throughput for $\mathrm{BO}=\mathrm{SO}=0$, parameterized on different $\mathrm{BE}$ and $\mathrm{L}$.

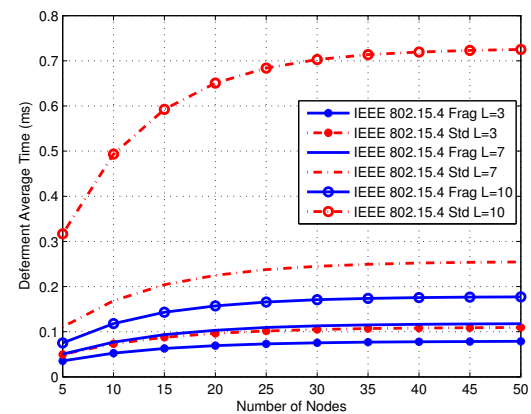

(a) $\mathrm{SO}=0$ and $\mathrm{BE}=2$

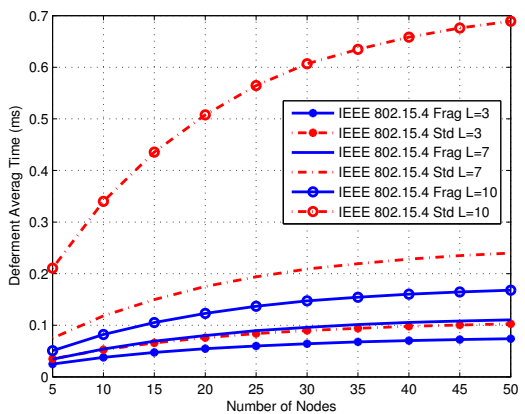

(b) $\mathrm{SO}=0$ and $\mathrm{BE}=3$

Fig. 10 Deferment average time for $\mathrm{BO}=\mathrm{SO}=0$, parameterized on different $\mathrm{BE}$ and $\mathrm{L}$

802.15.4 standard. In fact, the deferment's average time is slightly improved when $L=30$ bytes. However, the time deferment increases with increasing the size of the packet and when $L=10$ bytes, the fragmentation gives the best results.

\section{D) Access delay}

Figure 11 shows the access time values of successful transmissions. In our case, the network is saturated. So, when the value of $B E$ is equal to 2, the risk of finding the channel busy and having collisions increases, which causes additional delays before a successful packet transmission. We see in subfigures 11(a) that the time required for a successful packet transmission of the 50 nodes is less than 3 seconds (i.e. AccessDelay $<3 s)$. However, with $B E$ equal to 3 , the access time to successfully transmit all the packets of the $50^{\text {th }}$ considered nodes is less than 0.4 seconds, as illustrated in subfigures 11(b).

This difference is mainly caused by collisions and deferred transmissions for the following superframe. Note that, the proposed fragmentation mechanism clearly allows the reduction of the access delay in both cases of $\mathrm{BE}$ values. The obtained results are improved when the risk of collision is minimal as it can be seen from figure 11(b). 


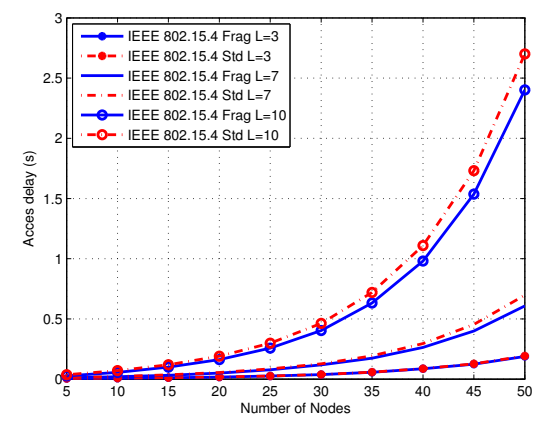

(a) $\mathrm{SO}=0$ and $\mathrm{BE}=2$

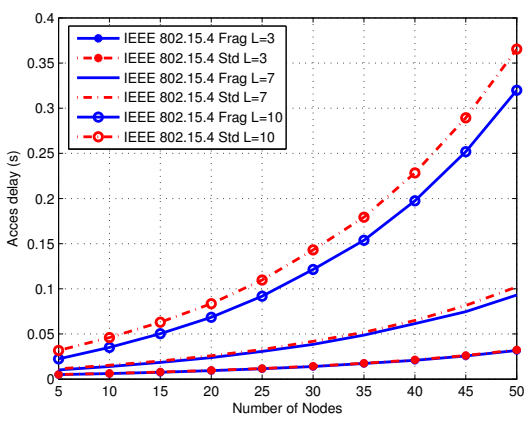

(b) $\mathrm{SO}=0$ and $\mathrm{BE}=3$

Fig. 11 Access delay for $\mathrm{BO}=\mathrm{SO}=0$, parameterized on different $\mathrm{BE}$ and $\mathrm{L}$

\section{E) Energy conservation}

To illustrate the energy conservation that can be realized with the new fragmentation mechanism when applying the NAV technique, we present the analytical results in the figures above. The energy consumption results of $N=10$ nodes and $N=30$ nodes using slotted CSMA/CA with fragmentation and slotted CSMA/CA with fragmentation and NAV technique are depicted in figure 12.

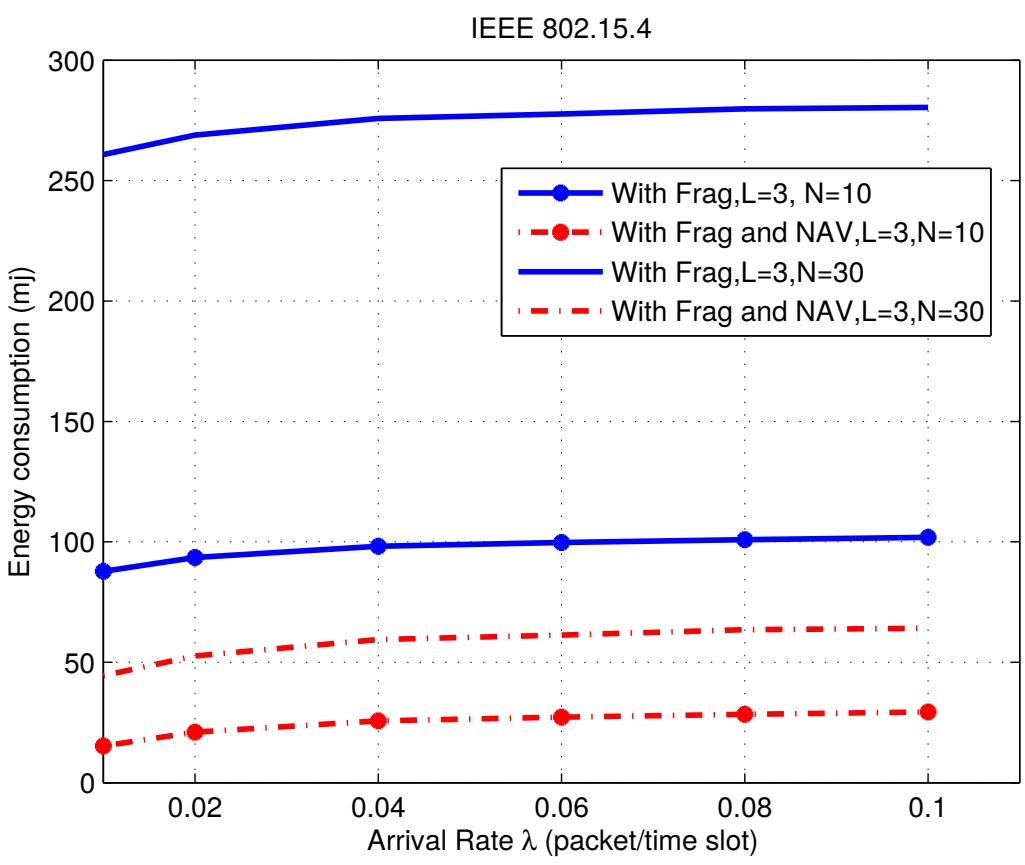

Fig. 12 Energy vs $\lambda$ arrival rate for the numbre of nodes $(\mathrm{N}=10$ and $\mathrm{N}=30)$ 
In the figure 12 we show that, when the packets' arrival rate increases, the power consumption increases too. However, when we introduce the NAV technique into the fragmentation mechanism, the energy consumption is less than IEEE 802.15.4 with only the fragmentation mechanism. Besides, when there is a high number of nodes $(\mathrm{N}=30)$, the energy conservation with the NAV technique is more significant than when having 10 nodes. These difference can be interpreted by the high number of nodes being in sleep mode in the beginning of the superframe, in case a node reserved the channel. The figure 13 shows another case of data conservation. So, when the length of the data frame to be transmitted is big, the energy consumption is high.

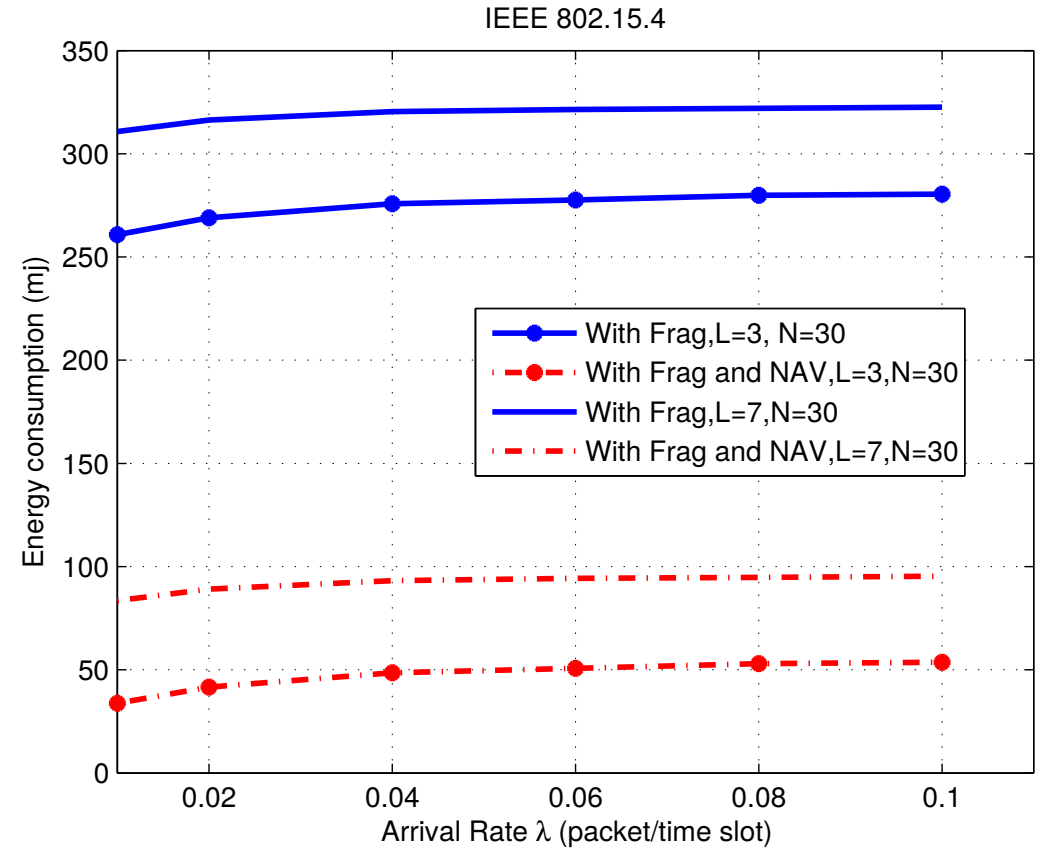

Fig. 13 Energy vs $\lambda$ arrival rate for different length of data frame

\section{Conclusion}

In this paper, we proposed to introduce a network allocation vector (NAV) technique in the data fragmentation mechanism. The NAV information is transmitted within the beacon frame to allow PAN nodes to conserve their energy for the NAV remaining period. We also show that applying data fragmentation mechanism under saturated traffic conditions is beneficial for the IEEE 802.15.4 performances. To evaluate the effectiveness of the proposed approach, we presented a Markov chainbased model of the slotted CSMA/CA protocol, which includes a fragmentation mechanism. Using the proposed model, we have evaluated the system performance. 
We have shown that the fragmentation mechanism allows having improved results compared to the original slotted CSMA/CA protocol in terms of bandwidth occupation, access delay, throughput and collision avoidance. We, also, show that the introduction of the NAV technique reduces energy consumption in the network compared with the traditional version of data fragmentation.

\section{References}

1. M. Atmani, D. Aïssani, and Y. Hadjadj-Aoul, Applying data fragmentation in IEEE 802.15.4: modeling and analysis under unsaturated traffic, In Proceedings of the VECoS'2014 [8th International Workshop on Verification and Evaluation of Computer and Communication Systems], Bejaia, September 2014, pp.121-130. ISBN : 978-9931-9140-3-7. http://ceurws.org/ vol-1256.

2. K. Sohrabi, J. Gao, V. Ailawadhi and G. J. Pottie, Protocols for Self-Organization of a Wireless Sensor Network, 37th Allerton Conference on Communication, Computing and Control, vol.7, no. 5, pp. 16-27 (1999).

3. C. Y. Lee, H. I. Cho, G. U. Hwang, Y. Doh, N. Park. Performance modeling and analysis of IEEE 802.15.4 slotted CSMA/CA protocol with ACK mode, International Journal of Electronics and Communications, vol. 65, no. 2, pp. 123-131 (February 2011).

4. IEEE Std 802.15.4. Part 15.4, Wireless Medium Access Control (MAC) and Physical Layer (PHY) Specifications for Low-Rate Wireless Area Sensor Networks (WPANs) (2003).

5. IEEE std 802.15.4, Part 15.4, Wireless Medium Access Control (MAC) and Physical Layer (PHY) Specifications for Low-Rate Wireless Personal Area Networks (WPANs) (2006).

6. S. Pollin, M. Ergen, S.C. Ergen, B. Bougard, L.V. Perre, I. Moerman, A. Bahai, P. Varaiya, and F. Catthoor, Performance Analysis of Slotted Carrier Sense IEEE 802.15.4 Medium Access Layer, IEEE Transactions on Wireless Communications, vol. 7, no. 9, pp. 3359-3371 (September 2008).

7. P. Park, P. Di Marco, P. Soldati, C. Fischione, and K.H. Johansson, A Generalized Markov Chain Model for Effective Analysis of Slotted IEEE 802.15.4, IEEE 6th International Conference on Mobile Ad Hoc and Sensor System (MASS), pp. 130-139 (Octobre 2009).

8. R. K. Patro, M. Raina, V. Ganapathy, M. Shamaiah, C. Thejaswi, Analysis and improvement of contention access protocol in IEEE 802.15.4 star network. The Fourth IEEE International Conference on Mobile Ad-hoc and Sensor Systems, Pisa, Italy (October 2007).

9. C. M. Wong, B. H. Lee, An Improvement of Slotted CSMA/CA Algorithm in IEEE 802.15.4 Medium Access Layer, Wireless Personal Communications, vol. 63, no. 4, pp.807-822 (April 2012).

10. S. U. Rehman, S. Berber, A. Swain. Performance Analysis of CSMA/CA Algorithm for Wireless Sensor Network, in TENCON 2010 - 2010 IEEE Region 10 Conference, pp. 20122017 (november 2010).

11. S. U. Rehman, F. A. Bhatti, M. Y. Iqbal, Z. Sabir. Modeling the Impact of Deferred Transmission in CSMA/CA Algorithm for IEEE 802.15.4 using Markov Chain Model, in Multitopic Conference (INMIC), 2011 IEEE 14th International, pp. 334-339 (december 2011).

12. Jongwon Yoon; Hyogon Kim; Jeong-Gil Ko; , "Data Fragmentation Scheme in IEEE 802.15.4 Wireless Sensor Networks," Vehicular Technology Conference, 2007. VTC2007Spring. IEEE 65th , vol. no., pp.26-30, 22-25 April 2007.

13. IEEE Part 11, Wireless LAN medium access control (MAC) and physical layer (PHY) specifications. IEEE Std 802.11 (2007).

14. M. Yazid, L. Bouallouche-Medjkoune, D. Aissani, L. Ziane-Khodja, Analytical analysis of applying packet fragmentation mechanism on IEEE 802.11b DCF network in non ideal channel with infinite load conditions, Wireless Network, Vol. 20, no. 5, pp. 917-934 (October 2013).

15. T. Li, Q. Ni, D. Malone, D. Leith, Aggregation With Fragment Retransmission for Very High-Speed WLANs, IEEE/ACM transactions on networking, vol. 17, no. 2, pp. 591-604 (April 2009).

16. J. Yoon, H. Kim and J. G. Ko, Data Fragmentation Scheme in IEEE 802.15.4 Wireless Sensor Networks, IEEE 65th Vehicular Technology Conference, Dublin, Ireland, pp. 26-30 (April 2007). 
17. N. Fourty, A. V. D. Bossche, T. Val, An advanced study of energy consumption in an IEEE 802.15.4 based network: Everything but the truth on 802.15.4 node lifetime, Computer Communications, vol. 35, no. 14, pp. 1759-1767 (August 2012).

18. IEEE Std 802.15.4a, Part 15.4: Wireless Medium Access Control (MAC) and Physical Layer (PHY) Specifications for Low-Rate Wireless Personal Area Networks (WPANs), 2007.

19. IEEE Std 802.15.4c, Part 15.4: Wireless Medium Access Control (MAC) and Physical Layer (PHY) Specifications for Low-Rate Wireless Personal Area Networks (WPANs), 2009. 20. IEEE Std 802.15.4d, Part 15.4: Wireless Medium Access Control (MAC) and Physical Layer (PHY) Specifications for Low-Rate Wireless Personal Area Networks (WPANs), 2009. 21. IEEE Std 802.15.4f, Part 15.4: Low-Rate Wireless Personal Area Networks (LR-WPANs), 2012 .

22. G. Bianchi, Performance Analysis of the IEEE 802.11 Distributed Coordination Function, IEEE journal on selected areas in communications, vol. 18, no. 3 (March 2000).

23. H. Wen, C. Lin, Z.J. Chen et al, An Improved Markov Model for IEEE 802.15.4 Slotted CSMA/CA Mechanism, journal of computer science and technology vol. 24, no. 3, pp.495-504 (May 2009).

24. T. J. Lee, H. R. Lee and M. Y. Chung, MAC Throughput Limit Analysis of Slotted CSMA/CA in IEEE 802.15.4 WPAN. IEEE communications letters, vol. 10, no. 7, pp. 561563 (July 2006).

25. M. Kohvakka, M. Kuorilehto, M. Hnnikinen and T. D. Hmlinen, Performance Analysis of IEEE 802.15.4 and ZigBee for Large-Scale Wireless Sensor Network Applications, PEWASUN, Malaga, Spain (2006). 